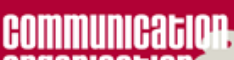
Organllotidn-

\section{Communication et organisation}

Revue scientifique francophone en Communication organisationnelle

59 | 2021

La communication constitutive des organisations : émergence et innovations

\title{
LE ROULLEY, Simon et UHEL, Mathieu (dir.), 2020. Chercheur.e's critiques en terrains critiques
}

Lormont : Éditions Le bord de l'eau

\section{Alexia Cappuccio}

\section{OpenEdition}

\section{Journals}

Édition électronique

URL : https://journals.openedition.org/communicationorganisation/9619

DOI : 10.4000/communicationorganisation.9619

ISSN : $1775-3546$

Éditeur

Presses universitaires de Bordeaux

Édition imprimée

Date de publication : 1 juin 2021

Pagination : 293-297

ISSN : $1168-5549$

Référence électronique

Alexia Cappuccio, «LE ROULLEY, Simon et UHEL, Mathieu (dir.), 2020. Chercheur.e.s critiques en terrains critiques », Communication et organisation [En ligne], 59 | 2021, mis en ligne le 01 juin 2021 , consulté le 02 décembre 2022. URL : http://journals.openedition.org/communicationorganisation/ 9619 ; DOI : https://doi.org/10.4000/communicationorganisation.9619 


\section{LE ROULLEY, Simon et UHEL, Mathieu (dir.), 2020. Chercheur.e.s critiques en terrains critiques. \\ Lormont : Éditions Le bord de l'eau \\ Par Alexia Cappuccio}

L'ouvrage collectif que nous souhaitons analyser ici s'intitule "Chercheur.e.s critiques en terrains critiques». Paru en 2020, il a été dirigé par Simon Le Roulley et Mathieu Uhel, respectivement docteur en sociologie et chercheur au Laboratoire d'Économie et de Sociologie du Travail (Aix-Marseille Université) et Maître de conférences en géographie sociale (Université de Caen). Dans un contexte où la question des thèses et des travaux académiques qualifiés d' "islamo-gauchistes " prend une place importante dans le débat public, cette œuvre offre un éclairage pertinent sur la recherche en sciences humaines et sociales (SHS), au prisme de huit contributions de chercheurs français et internationaux issus principalement des disciplines de la sociologie et de la géographie sociale. 
L'ouvrage présente une posture assumée des chercheurs vis-à-vis de leur implication personnelle et émotionnelle dans leurs travaux. Leur subjectivité est présentée comme une condition de la pluralité et de la diversité des recherches, en ce qu'elle permet la découverte de nouveaux sujets et objets de recherche ainsi que l'accès à des terrains privilégiés ou confidentiels. L'ouvrage traite également des approches qualitatives présentées dans les différents chapitres. Les contributeurs développent une réflexion sur les interrelations entre recherche et militantisme, envisagés comme des vases communicants dans une perspective de "co-construction et de reconstruction du savoir" (Kevin Cambervelle, Thomas Guyonnet, Anaïs Ousseni et Pauline Picot, p. 165), prenant en compte les expériences - voire les expertises - des enquêtés. La légitimité de ces enquêtes et de ces chercheurs " critiques " est assurée par la pratique de la réflexivité. Au prisme de ces trois arguments, l'ouvrage présente en filigrane une façon d'aborder la recherche en SHS qui differe de l'approche dominante objectivante, héritée des sciences dites "dures".

\section{La subjectivité des chercheurs, garante de la pluralité et de la diversité de la recherche}

Cet ouvrage a pour objectif de proposer une autre vision de la recherche que celle appliquée majoritairement aujourd'hui dans de nombreuses disciplines : démarches hypothético-déductives, mise à distance impérative du chercheur vis-à-vis de son sujet de recherche, objectivation des résultats... Les contributeurs ont ici souhaité décrire une approche qui consiste, pour eux, à se saisir de leurs personnalités et expériences singulières, afin de découvrir et de développer certains sujets, objets, terrains de recherches.

Les contributions font état de l'attachement profond des chercheurs à leurs sujets et objets de recherche. Cette dimension est particulièrement prégnante car la quasitotalité des contributions aborde des thématiques liées à ce que l'on pourrait décrire comme des injustices sociales, ou qui traitent de rapports inégaux de domination (Nicolas Bautès et Marcos Burgos, p. 127 ; Mathieu Uhel, pp. 146-147). Qu'il s'agisse des luttes étudiantes contre les réformes du travail ou de l'éducation (Renaud Lariagon), de l'accès à l'enseignement supérieur (Julie Le Mazier et Leïla Frouillou), ou bien de sujets tels que l'étude des processus de racisation et des mouvements intersectionnels (Kevin Cambervelle, Thomas Guyonnet, Anaïs Ousseni et Pauline Picot), en passant par des recherches sur le processus de gentrification des quartiers sensibles et sur les logiques de résistance qui s'y développent par la suite (Romain Geffrouais ; Florian Opillard), tous ces chercheurs ont puisé dans leurs sensibilités et leurs expériences pour développer leurs travaux.

Le chapitre d'Irène Pereira (pp. 47-63) constitue un exemple de cette posture spécifique. L'auteure a développé ce qu'elle qualifie "d'auto-ethnobiographie » dans le but de concilier vie professionnelle (précaire pendant un temps), vie privée et activité 
de recherche. Sa vie et sa trajectoire sociale, ses perceptions, sont donc à la base de son travail sur les conflits d'usage autour d'un lieu alternatif à Orléans, travail qui, selon elle, était le reflet «d'une situation sociale» (p. 50) : la sienne.

Cette sensibilité et ces expériences de vie constituent donc la condition même de l'émergence de ces enquêtes "critiques» et engageantes pour le chercheur. La contribution de Renaud Lariagon l'illustre parfaitement : l'auteur a engagé ses premiers travaux de recherche en géographie sociale sur les enjeux de territorialisation des luttes étudiantes, en raison de son passé de militant au cours de ses études supérieures. Le constat est similaire pour la recherche engagée sur la réforme de l'accès à l'enseignement supérieur, conduite par deux sociologues inscrites dans ce terrain particulier de l'éducation en tant que professeure principale en terminale et nouvelle Maîtresse de conférence. Il en va de même pour la contribution collective sur les mouvements intersectionnels dont certains des auteurs étaient issus. Dans le cas de cette dernière contribution, l'appartenance à la communauté étudiée a été une condition nécessaire pour accéder au terrain et recueillir des données.

La subjectivité des chercheurs est donc pensée, dans l'ouvrage, comme représentant un élément nécessaire à la diversité et à la pluralité de la recherche en SHS. Les contributions montrent bien que le choix de certains sujets, par ailleurs souvent délaissés par les financements publics (Irène Pereira ; Mathieu Uhel), ne peut s'opérer qu'à la condition que le chercheur dispose d'une sensibilité et d'un intérêt préalables. D'autre part, certains terrains se ferment lorsque les chercheurs n'en sont pas issus, comme le démontre la contribution de Kevin Cambervelle, Thomas Guyonnet, Anaïs Ousseni et Pauline Picot. Mais l'implication émotionnelle revendiquée par les chercheurs, ajoutée à la sensibilité des terrains abordés, induit la nécessité de mener une réflexion sur les liens entre recherche et militantisme.

\section{Recherche engagée et militantisme : un apport mutuel légitimé par la pratique de la réflexivité}

Certaines des contributions explorent les liens entre recherche et militantisme, et en interrogent la scientificité. L'engagement militant peut conduire à explorer des terrains spécifiques et parfois fermés. C'est le cas de Julie Le Mazier et Leïla Frouillou avec leur recherche "engagée contre Parcoursup " adossée à leur appartenance à un syndicat. Dans cette configuration, l'engagement militant a nourri le choix des sujets de recherche et a conditionné l'accès à des terrains plus ou moins fermés, tout en conférant aux chercheurs une position privilégiée, mais souvent renégociée au cours de l'enquête, pour recueillir des données (Nicolas Bautès et Marcos Burgos ; Romain Geffrouais ; Mathieu Uhel).

Sans être forcément engagés préalablement dans leurs recherches, d'autres contributeurs ont travaillé sur des terrains qualifiés de "critiques" ou de "sensibles", 
et sur des groupes sociaux traversés par des logiques militantes. Les chercheurs ont parfois dû, comme cela a été le cas entre autres pour Romain Geffrouais ou Florian Opillard, " mettre la main à la pâte » et lutter aux côtés de leurs enquêtés pour accéder et maintenir leur position au sein du terrain. Cela a toutefois eu pour conséquence de brouiller la frontière entre leur posture de chercheur et leur implication, soulevant ainsi des questionnements méthodologiques.

Un autre argument mis en exergue dans l'ouvrage est que le militantisme et la recherche se "nourrissent mutuellement" (Florian Opillard, p. 66). Le champ scientifique s'appuie sur les expériences singulières vécues par les membres des cercles militants pour nourrir la recherche de questionnements nouveaux. La recherche peut en retour produire du savoir pour alimenter les luttes, voire des outils pour aider les militants et les minorités à s'émanciper des mécanismes de domination (Romain Geffrouais, p. 77). De plus, le croisement de ces deux univers permet de proposer un éclairage particulier sur des sujets " chauds " (tels que le racisme ou les mouvements féministes intersectionnels) parfois minimisés dans les travaux produits par les chercheurs non issus de ces groupes minoritaires.

Cette posture singulière, à la croisée des deux mondes que sont la recherche académique et le militantisme, est perçue par les contributeurs comme une opportunité de déplacer le prisme d'analyse du chercheur du vertical, c'est-à-dire "surplombant" (Julie Le Mazier et Leïla Frouillou, p. 46) à une vision plus horizontale, en travaillant " avec" et non "sur» les terrains et groupes sociaux étudiés (Renaud Lariagon, p. 26). Cependant, la nécessité de ne pas produire des savoirs " trop militants» (Nicolas Bautès et Marcos Burgos, p. 128) est reconnue par une grande partie des contributeurs. C'est pourquoi ils proposent le concept de "réflexivité " comme garante de la légitimité de ces recherches engagées ou militantes.

La réflexivité est définie par Blanchet (2009, p.145)1 comme "une démarche par laquelle je m'interroge moi-même (comme je me regarde dans un miroir) et où je suis interrogé par autrui (qui me renvoie ainsi une image) sur mes propres actes et mes propres discours". Il s'agit, pour un chercheur, de s'interroger sur son rapport à son sujet. Blanchet (2009, pp.146-147) présente la réflexivité comme un outil permettant d'admettre qu'aucune science n'est neutre et objective, et comme un moyen de "réhabiliter", d' "humaniser » et de "socialiser " les recherches notamment ethnographiques, interprétatives et qualitatives, telles que celles présentées dans le recueil collectif analysé ici.

Les auteurs reconnaissent que leur implication personnelle et émotionnelle dans leur terrain demande un surcroît de réflexivité (Julie Le Mazier et Leïla Frouillou).

1- Blanchet, P. (2009). La réflexivité comme condition et comme objectif d'une recherche scientifique humaine et sociale. Cahiers de sociolinguistique, 2009/1 n 14 pp. 145-152, Presses universitaires de Rennes (http://www.cairn.info/revue-cahiers-de-sociolinguistique-2009-1-page-145.htm) 
Cette réflexivité est aussi présentée comme un moyen d'éviter l'objectivation des travaux académiques, qui se manifeste notamment par le primat accordé aux approches positivistes. L'enjeu est aussi d'éviter une forme d'autocensure des chercheurs souhaitant se pencher sur des terrains sensibles (Florian Opillard : 99), alors que l'enseignement universitaire continue de sensibiliser les étudiants aux « dangers de l'implication affective» dans les recherches académiques (Simon Le Roulley : 171).

Pour résumer, l'ouvrage tend à démontrer que les recherches engagées ou militantes - à condition qu'elles soient légitimées par une rigueur sans faille du chercheur et sa pratique de la réflexivité - sont essentielles pour plusieurs raisons. Tout d'abord, elles permettent l'exploration de sujets, d'objets et de terrains de recherche dont personne ne se serait saisi, à l'exception de ceux qui en sont issus. De plus, l'engagement du chercheur constitue souvent la condition de l'accès au terrain. La recherche et le militantisme sont envisagés ici comme deux mondes sociaux, parfois décrits comme poreux, qui peuvent s'enrichir l'un l'autre de leurs savoirs spécifiques, bien qu'il soit admis par les contributeurs que les enjeux des deux champs soient différents.

La légitimation de ce type de recherches engagées, relevant des théories critiques ou des théories de l'action située, se fait à la condition que les chercheurs mettent en œuvre le concept de réflexivité. Finalement, la réflexivité, comme Blanchet la conceptualise (2009 : 146) et telle qu'elle est présentée dans l'ouvrage, permet d'envisager les sciences comme étant non-neutres, contrairement au paradigme dominant issu des sciences dites " dures ". En effet, Simon Le Roulley (p.173) déclare dans la conclusion générale de l'ouvrage : "Quill soit déclaratif ou non, il y a donc un engagement inhérent à toute science». Selon lui, l'enjeu est donc de se saisir de cet engagement, au prisme de la réflexivité, pour conduire des recherches librement, sans autocensure, et sans crainte de voir son travail disqualifié, discrédité, soit vis-à-vis d'un positionnement "scientifico-politique " (Mathieu Uhel : 146), soit au regard des productions de savoirs des sciences dites « exactes ».

Référence : LE ROULLEY, Simon et UHEL, Mathieu (dir.), 2020. Chercheur.e.s critiques en terrains critiques. Lormont : Éditions Le bord de l'eau. ISBN 978-235687-719-2, 24€.

Alexia Cappuccio est doctorante à l'IMSIC et chargée d'enseignement à l'École de Journalisme et de Communication d'Aix-Marseille (EJCAM). Ses travaux portent sur la sociologie du journalisme, la sociologie de la production de l'information et sur les médias en tant qu'organisations économiques. Elle s'intéresse aux effets de l'environnement et des conditions de travail spécifiques des journalistes de Radio France sur leurs vécus, leurs perceptions de leur métier et de leurs rôles, ainsi que sur leurs productions. 